\title{
Novel Off-Pump Coronary Artery Bypass Grafting in Right Coronary Artery Stenosis
}

\author{
Masafumi Hashimoto ${ }^{1}$, Kenji Mogi ${ }^{2}$, Manabu Sakurai ${ }^{1}$, Kengo Tani ${ }^{2}$, Shuntaro Ito $^{1}$, and \\ Yoshiharu Takahara ${ }^{3}$ \\ ${ }^{1}$ Funabashi Municipal Medical Center \\ ${ }^{2}$ Funabashi Municipal Medical Center Heart and Vascular Institute \\ ${ }^{3}$ Affiliation not available
}

August 28, 2020

\begin{abstract}
A 77-year-old man with diabetes, dyslipidemia, and a smoking history presented with asymptomatic gross hematuria and left hydronephrosis. Computed tomography (CT) angiography revealed a left ureteral tumor and abdominal aortic aneurysm. Cardiac catheterization revealed right coronary artery (RCA) stenosis. First, a left nephroureterectomy was performed via a midline abdominal incision. To achieve minimal invasiveness, a median sternotomy was avoided, and off-pump coronary artery bypass grafting of the RCA was performed with the great saphenous vein graft, using the left renal artery as the graft inflow. Y-grafting was subsequently performed. Without any postoperative complications, CT angiography confirmed graft patency. This procedure has potential use for removing ureteral tumors by surgeons and clinicians in clinical settings.
\end{abstract}

\section{Novel Off-Pump Coronary Artery Bypass Grafting in Right Coronary Artery Stenosis}

Short Running Title: Off-pump grafting in RCA

Masafumi Hashimoto, $\mathrm{MD}^{1}$, Kenji Mogi, $\mathrm{MD}, \mathrm{PhD}^{1}$, Manabu Sakurai, MD, $\mathrm{PhD}^{1}$

Kengo Tani, $\mathrm{MD}^{1}$, Shuntaro Ito, $\mathrm{MD}^{1}$, and Yoshiharu Takahara ${ }^{1}$

${ }^{1}$ Division of Cardiovascular Surgery, Funabashi Municipal Medical Center Heart and Vascular Institute, 1-21-1 Kanasugi, Funabashi, Chiba, Japan

Corresponding author: Masafumi Hashimoto, MD

Division of Cardiovascular Surgery, Funabashi Municipal Medical Center Heart and Vascular Institute, 1-21-1 Kanasugi, Funabashi, Chiba, Japan

E-mail address: m.hashimoto0610@gmail.com

Zip code: $273-8588$

Phone number: +81-47-438-3321

\section{Funding:none}

\section{Meetings:none}

A 77-year-old man with diabetes, dyslipidemia, and a history of smoking presented with asymptomatic, gross hematuria and left hydronephrosis. Computed tomography (CT) angiography revealed a left ureteral tumor 
$(25 \times 18 \mathrm{~mm})$ and an abdominal aortic aneurysm $(76 \times 73 \mathrm{~mm})$ (Figure 1). Cardiac catheterization revealed right coronary artery (RCA) stenosis (Figure 2). Bone scintigraphy revealed no bone metastasis.

First, a left nephroureterectomy was performed via a midline abdominal incision. For minimal invasiveness, a median sternotomy was avoided, and off-pump coronary artery bypass grafting of the RCA was performed with the great saphenous vein graft, using the left renal artery as the graft inflow. The arterial grafts (employing the radial artery) were insufficiently long. Y-grafting was subsequently performed.

Post-surgery, the patient experienced no complications and was discharged on the $25^{\text {th }}$ postoperative day. Postoperative CT angiogram confirmed graft patency (Figure 3). The patient provided informed consent for publishing this case report.

Pathological examination of the removed left kidney and ureter revealed a non-invasive low-grade papillary urothelial carcinoma. Urothelial carcinoma reportedly metastasizes through the intravenous route. Therefore, this method supposedly has no adverse effects on cancerous metastases. For a transdiaphragmatic approach, a method using the gastroduodenal artery as the inflow site has been reported. ${ }^{1,2}$ This procedure has potential use for removing ureteral tumors by surgeons and clinicians in clinical settings.

\section{Conflict of interest}

The authors have no conflicts of interest to declare.

\section{Author contributions}

None

\section{Figure legends}

Figure 1: CT angiogram reveals a left ureteral tumor $(25 \times 18 \mathrm{~mm})$ (arrow) and abdominal aortic aneurysm $(76 \times 73 \mathrm{~mm})$

Figure 2: Cardiac catheterization reveals RCA stenosis (\#2: 99\%) (arrow)

Figure 3: The postoperative CT angiogram confirms graft patency. The SVG reaches the RCA (posterior descending artery) via the diaphragm, using the left renal artery (Lt.RA) as the graft inflow.

\section{References}

1. Takahashi K, Minakawa M, Kondo N, Oikawa S, Hatakeyama M. Coronary artery bypass surgery by the transdiaphragmatic approach. Ann Thorac Surg 2002;74:700-703.

2. Norihiro K, Kenji T, Masahito M, Shigeru O, Masaharu H. Coronary artery bypass via diaphragmatic approach with free graft. Ann Thorac Surg 2002;74:939-940. 


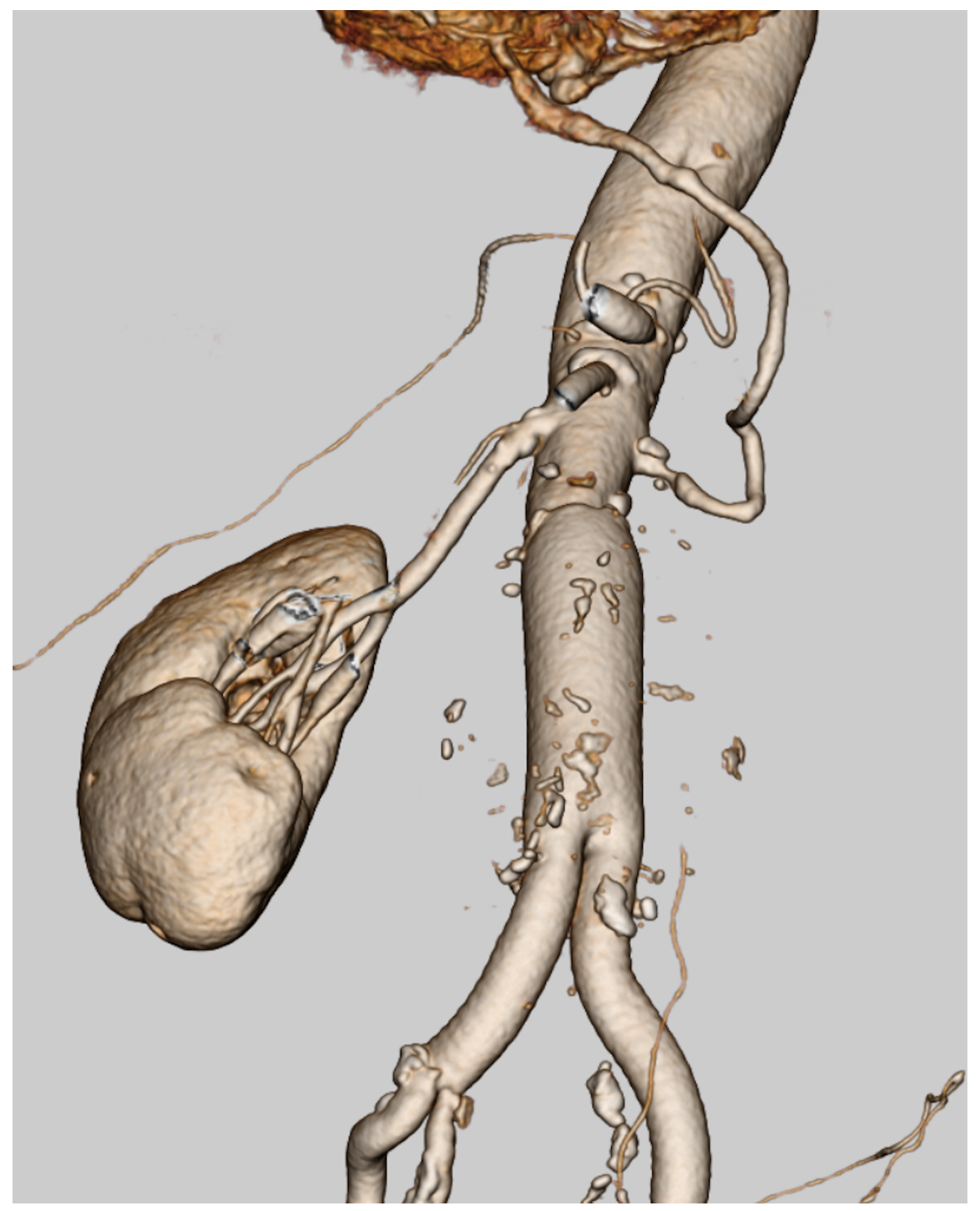




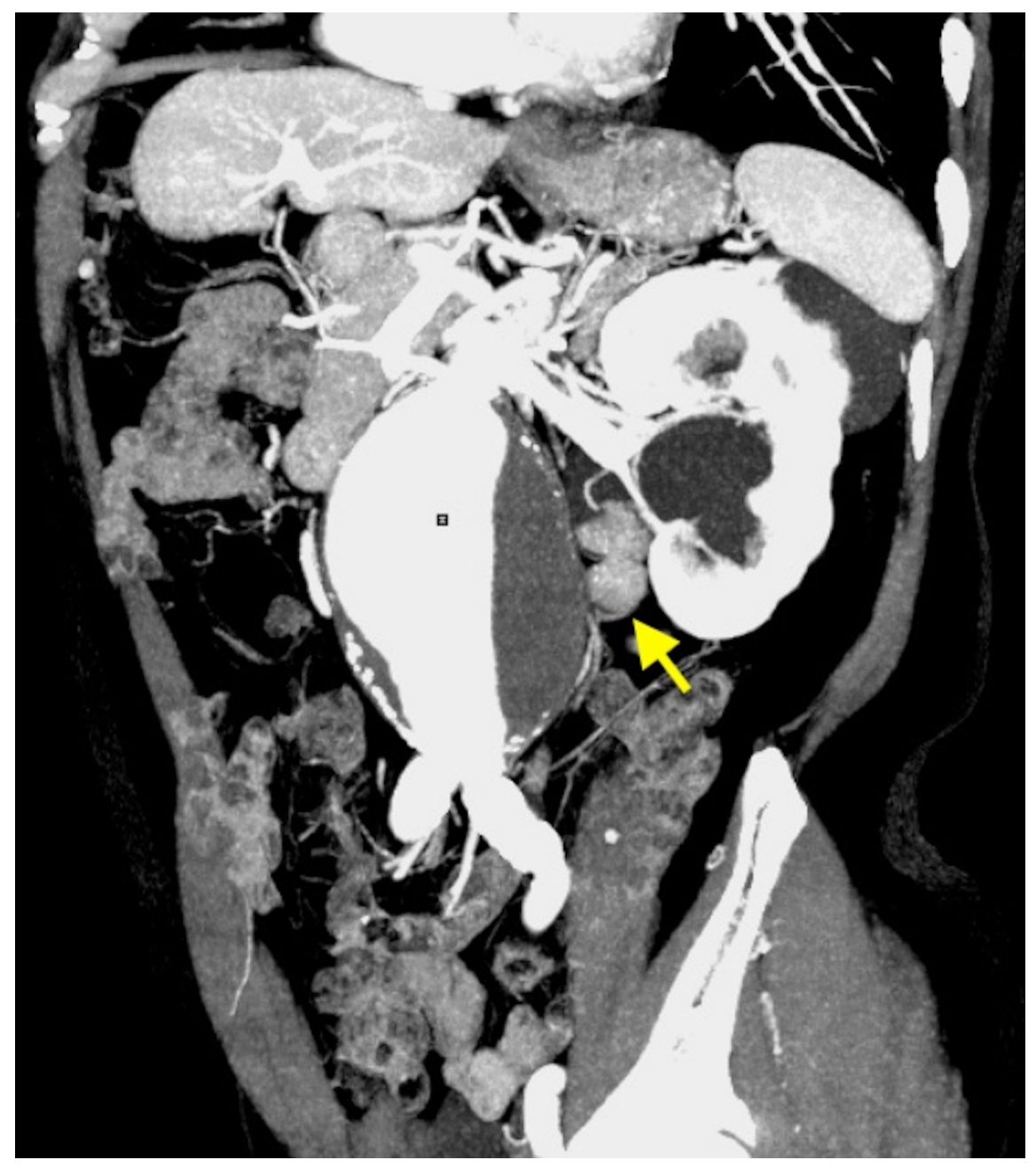




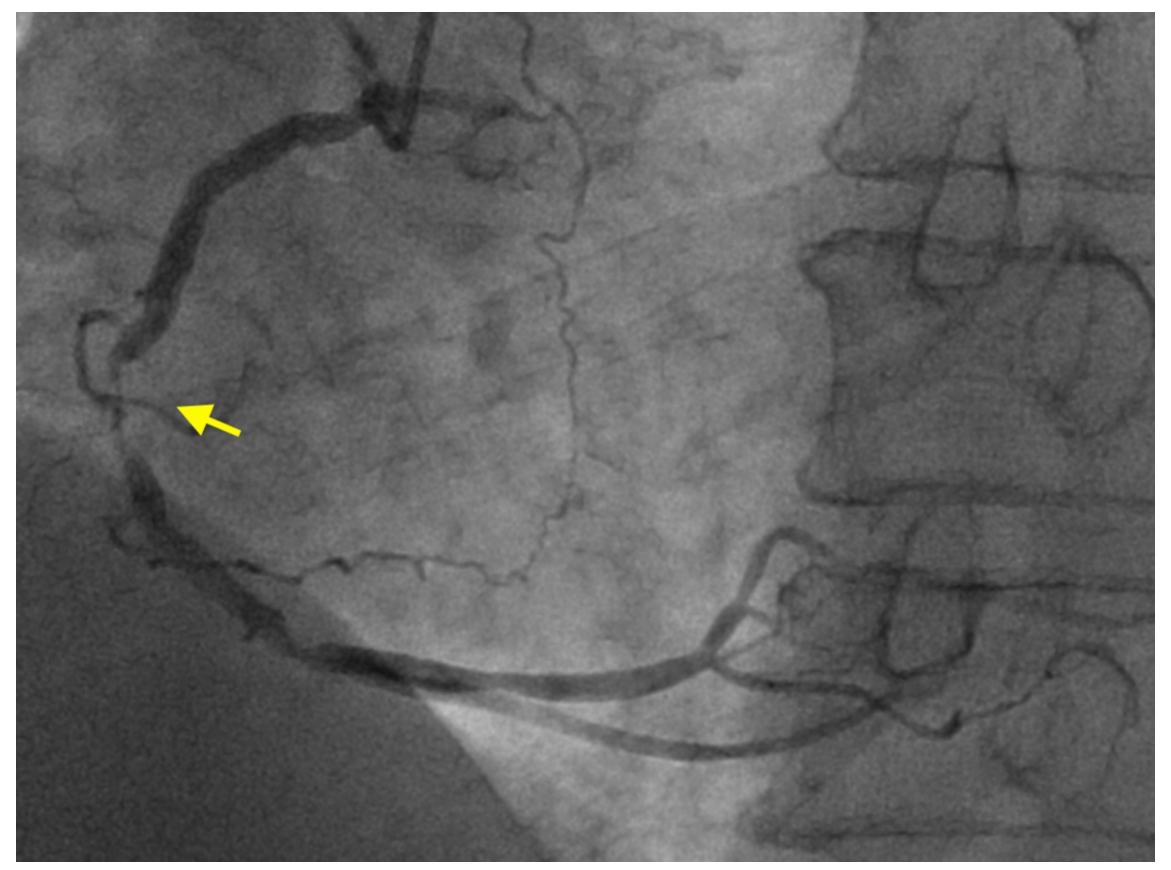

